\title{
Do we remember templates better so that we can reject distractors better?
}

\author{
Jason Rajsic ${ }^{1}$ Geoffrey F. Woodman ${ }^{1}$
}

Published online: 1 April 2019

(C) The Psychonomic Society, Inc. 2019

\begin{abstract}
Feature Integration Theory proposed that attention shifted between target-like representations in our visual field. However, the nature of the representations that determined what was target like received less specification than the nature of the attention shifts. In recent years, visual search research has focused on the nature of the memory representations that we use to guide our shifts of attention. Sensitive measures of memory quality indicate that the template representations are remembered better than other, merely maintained, memories. Here we tested the hypothesis that we prepare for difficult search tasks by storing a higher fidelity target representation in working memory than we do when preparing for an easy search task. To test this hypothesis, we explicitly tested participants' memory of the target color they searched for (i.e., the attentional template) versus another memory that was not used to guide attention (i.e., an accessory representation) following blocks of searches with easy-to-find targets (i.e., distractors were homogeneously colored) to blocks of searches with hard-to-find targets (i.e., distractors were heterogeneously colored). Although homogeneous-distractor searches required minimal precision for distractor rejection, we found that templates were still remembered better than accessories, just like we found in a heterogeneous-distractor search. As a consequence, we suggest that stronger memories for templates likely reflects the need to decide whether new perceptual inputs match the template, and not an attempt to create a better template representation in anticipation of difficult searches.
\end{abstract}

Keywords Visual search $\cdot$ Visual working memory $\cdot$ Attention: Selective

\section{Introduction}

While our world abounds with detailed visual information, successful behavior relies on our ability to focus on the taskrelevant pieces of information. Research on how we find and focus on task-relevant objects in a cluttered visual field was revolutionized with the publication of Treisman's Feature Integration Theory of Attention (FIT; Treisman \& Gelade, 1980). This theory made the bold claim that despite the wholly integrated subjective percepts we experience, "features come first in perception" (Treisman \& Gelade, 1980, p. 98). While FIT was a theory of perception, broadly construed, it had an especially large influence on studies of visual search. Indeed,

Jason Rajsic

jason.rajsic@vanderbilt.edu

1 Department of Psychology, Center for Integrative and Cognitive Neuroscience, Vanderbilt Vision Research Center, Vanderbilt University, PMB 407817, 2301 Vanderbilt Place,

Nashville, TN 37240-7817, USA it was the results of visual search experiments (Nakayama \& Silverman, 1986; Pashler, 1987; Wolfe, Cave, \& Franzel, 1989) that led to a revision of FIT 10 years later by Treisman and Sato (1990). Treisman's revised account acknowledged that scanning through displays of un-bound conjunctions was not strictly random. Although still fundamentally feature-based, our scans can exclude stimuli with irrelevant features when we search a display for a target.

In the years since, a great deal of research has been devoted to understanding the control processes that allow us to focus on task-relevant objects during search (Carlisle \& Woodman, 2011; Desimone \& Duncan, 1995; Kiyonaga, Egner, \& Soto, 2012; Olivers, Meijer, \& Theeuwes, 2006; Woodman, Vogel, \& Luck, 2001). Searching for a stimulus for the first time requires representing its features in working memory (van Moorselaar, Theeuwes, \& Olivers, 2016; Woodman, Carlisle, \& Reinhart, 2013). However, representing stimulus features in working memory is not the same as searching for a stimulus with these features. If we maintain multiple stimulus representations in working memory, but only need to look for one of those stimuli, visual attention can be effectively restricted to those stimuli 
matching just the sought after stimulus representation (Downing \& Dodds, 2004; Peters, Goebel, \& Roelfsema, 2008). Consequently, Olivers, Peters, Houtkamp, and Roelfsema (2011) proposed that the memory representations we use to guide attention - often known as search templates are maintained in a special state in visual working memory, and that memories not used to guide search are maintained as accessory items, in a state that cannot influence the settings of current priority maps (Zelinsky \& Bisley, 2015).

Recently, Rajsic, Ouslis, Wilson, and Pratt (2017) found that a consequence of assigning template status to a representation in working memory is that this memory can be reported with greater fidelity than an accessory memory. This was the case even when neither remembered color was encountered during search, suggesting that making a memory into a search template does not only prevent accessory items from interacting with visual attention, but shapes the memories themselves. Furthermore, templates were remembered better than accessories even on occasional trials where the search did not occur, consistent with the idea that this memory reweighting occurs in preparation for search and not during the search itself. Given that memory fidelity differed between templates and accessories, this measure could provide a behavioral index of the mental representations that allow searchers to selectively scan target-like items, as Treisman proposed (Treisman \& Sato, 1990). However, it is not clear from this previous work whether this improved memory for templates marks something special about search templates per se, or whether it reflects a more generic selection of internal information that is task-relevant (Myers, Stokes, \& Nobre, 2017; Souza \& Oberauer, 2016). If the memory advantage for templates is a consequence of shaping the template memory representation to more efficiently reject distractors in anticipation of performing search, then one can predict that its memory advantage over accessory items will only be observed in the context of search tasks that create sufficient competition for spatial attention.

As noted earlier, one hypothesized function of search templates is to guide search to stimulus locations that are worth searching, given that the features at that location are similar to the target templates (Duncan \& Humphreys, 1989; Wolfe et al., 1989; Zelinsky, 2008). We know that target representations can be used like this because search can be restricted to subsets of items in a display sharing a feature, reducing the effective search size (Egeth, Virzi, \& Garbart, 1984; Friedman-Hill \& Wolfe, 1995; Zohary \& Hochstein, 1989), and search is more efficient when targets share fewer features with distractors (Wolfe et al., 1989). It follows that more precise templates should enable a reduction in the effective set size of search.

Experiments that have manipulated the precision of search templates have indeed found a relationship between template precision and guidance. Hout and Goldinger (2015) had participants search for realistic objects and found that less precise templates resulted in more inefficient search. Template precision was manipulated in two ways: by including targets that matched a pictorial cue to varying extents (e.g., the exact mug cued or another mug that was cued, but was still the only mug in the display) and by comparing dual-target searches when the two sought-after targets were more or less visually similar. Both manipulations of template precision affected scan-paths, which were taken to indicate the strength of attentional guidance. Thus, increases in template precision do appear to increase the efficiency of search. It is therefore plausible that participants remember templates more precisely than accessory items because this allows for guidance to fewer candidate items during search. We will refer to this account as the adaptive-weighting hypothesis. This hypothesis states that representations of templates are strategically weighted over accessory memories to improve search efficiency. Specifically, this account predicts that when searchers know that targets are harder to find, they intentionally weight the storage of the template more heavily than the accessory in advance of each search, but do not weight the template more than accessory items when the target can be found without a template (i.e., because the target pops out).

Although improving the fidelity of a memory when it becomes a search template could serve the function of improving search efficiency, it could instead be a consequence of having to use a representation to make a decision, regardless of the perceptual load associated with the upcoming search. Preparing to make a decision about whether or not a stimulus matches one, but not another, memory representation requires some mechanism for focusing the decision on the correct stimulus-memory pair (Summerfield \& Koechlin, 2008). Simply preparing a memory to be compared with incoming perceptual inputs may be sufficient to produce memory benefits for the template memory, costs for the accessory memory, or both (Myers et al., 2017; Reinhart \& Woodman, 2014; Zokaei, Ning, Manohar, Feredoes, \& Husain, 2014). We will refer to this account as the recognition-weighting hypothesis. This hypothesis proposes that preparatory weighting of the template over accessory memory representations occurs because targets must be recognized based on a template, even if the target can be localized via unique physical salience (i.e., popping out), such that the benefit of weighting the template presumably lies in facilitating target recognition, once it has been localized, rather than more efficient localization of the target during search.

Recent research by Geng, DiQuattro, and Helm (2017) has directly shown that templates are indeed sharpened when distractors are more likely to be similar to the target, lending some support to the hypothesis that the template memory benefit is related to segregation of the target from concurrent distractors. One potentially important factor, though, is the consistency of target colors. 
Electrophysiological research has shown that repeatedly looking for the same target allows long-term memory to participate in visual search (Woodman et al., 2013). As such, it is possible that this improvement in template precision reported by Geng and colleagues resulted from repeated exposure to target and distractor color values such that the sharpening that was observed was of a long-term memory representation of the target. To rule out such an explanation in our experiments a new color was the target on every trial, and so any change in template precision must be due to cognitive control over the working memory representation of the target.

Experiment 1 was designed to test the hypothesis that templates are remembered better so that distractors can be rejected more effectively. We ran two between-subjects conditions: a heterogeneous search and a homogeneous search. Borrowing from the design of Rajsic et al. (2017), we had participants remember two colors on each trial. One was the target, which we call the template in following text, and the other was an item that they knew they would be tested as often, that we will call the accessory item in the following text (see Fig. 1). If templates are remembered better than accessories so that search guidance can be improved, then we expect that templates will be remembered better than accessory items in the heterogeneous condition, but not the homogenous condition. This is because when distractors are homogeneous, no guidance is necessary since the search target can be localized using bottom-up contrast signals alone (Bacon \& Egeth, 1994). On the other hand, if templates are remembered better because making any target discrimination decision entails a special cognitive state compared to just remembering an object, then both heterogeneous and homogenous searches will lead to a difference in memory quality between templates and accessories.

\section{Experiment 1}

\section{Methods}

\section{Participants}

Thirty participants volunteered for Experiment 1. All were recruited from Vanderbilt's online experiment system, participated in exchange for course credit, and provided informed consent before participating in procedures approved by the Vanderbilt University Institutional Review Board. Six participants were excluded from analysis for having either their search or their memory performance at chance (i.e., indistinguishable from chance in one or more conditions). Chance performance in the search was defined as accuracy below $58 \%$ in any condition (i.e., the $95 \%$ cutoff for a one-tailed binomial test with 100 observations and 50\% probability of success). Chance in the memory task was estimated using simulations. More specifically, we computed the standard deviation between 50 pairs of randomly chosen angles (i.e., the number of trials in a single condition) 10,000 times and chose the fifth percentile value as the cut-off for above-chance performance (given that lower standard deviation indicates high accuracy). Five participants in the heterogeneous search condition and one participant in the homogeneous search condition were excluded using these criteria. The same pattern of results was obtained with these participants included, but we preferred not to analyze data from participants who could, or did, not reliably complete both the search and memory components of the task. Data were collected until we obtained a sample of 12 participants in each condition after exclusion criteria were applied.
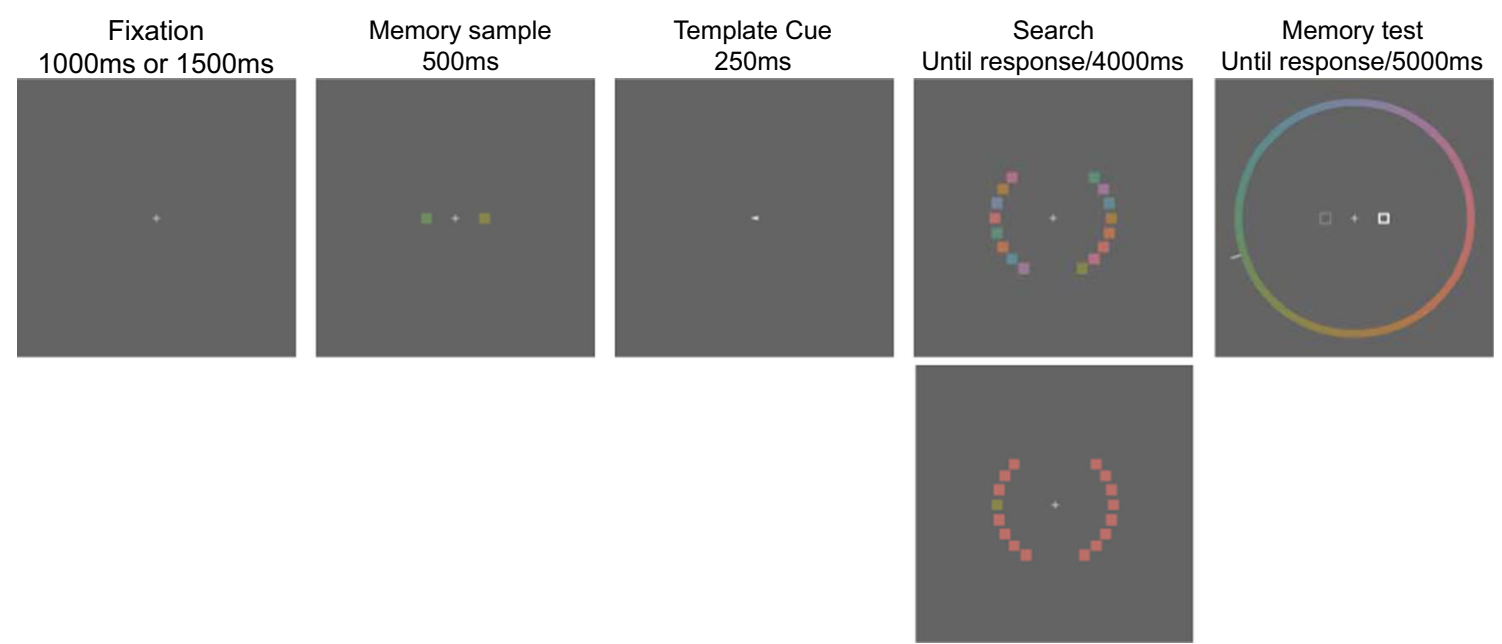

Fig. 1 An example trial sequence used in Experiment 1, showing both heterogeneous and homogeneous search examples. Not pictured are two fixation displays before and after the cue display indicating the target participants should search for (lasting $500 \mathrm{~ms}$ and 1,000 ms, respectively) 


\section{Stimuli and procedure}

Stimuli were presented to participants on an ASUS monitor and were generated using Matlab with the Psychophysics Toolbox (Kleiner, Brainard, \& Pelli, 2007). Participants viewed the stimuli from a distance of approximately $80 \mathrm{~cm}$. Participants entered responses using a standard USB keyboard.

Experimental stimuli on each trial comprised five kinds of displays, depicted in Fig. 1. The first display was a fixation display, consisting of a + in the middle of the screen $\left(0.8^{\circ}\right.$ in height and width) on a dark gray background for either $1,000 \mathrm{~ms}$ or $1,500 \mathrm{~ms}$. Next was the memory sample display. This display presented the two to-be-remembered colors for $500 \mathrm{~ms}$, one $3^{\circ}$ to the left of a fixation and one $3^{\circ}$ to the right of the fixation. Each was $1.1^{\circ}$ in height and width, and colored by sampling along the circumference of a circle in $L * A * B$ space, using Matlab's lab2rgb function, centered on $A=5$ and $\mathrm{B}=10$, with a radius of 25 , and a constant luminance value of $55 \%$. On each trial, ten equidistant colors were sampled, two of which were used as the memory stimuli, with the other eight reserved as potential distractor colors. After the memory sample array, a 500-ms fixation display preceded the cue array. Next, participants were shown a cue that indicated which of the two memorized items to use as a search template. The cue was a small arrow $\left(0.8^{\circ} \times 0.4^{\circ}\right)$ pointing to the left or right, lasting $250 \mathrm{~ms}$, with the arrowhead pointed to the location that had just contained the target color. We presented a fixation display for $1,000 \mathrm{~ms}$ before the search display onset. Search displays consisted of 16 squares $\left(1.1^{\circ} \times\right.$ $1.1^{\circ}$ ), arranged along the circumference of an imaginary circle, $6^{\circ}$ in radius. Search stimuli were drawn in two arcs, evenly spaced between $30^{\circ}$ and $150^{\circ}$ along the right half of the circle's circumference and between $210^{\circ}$ and $330^{\circ}$ along the left half of the circle's circumference.

There were three types of search arrays: template-present arrays, accessory-present arrays, and neither-present arrays. We created neither-present arrays first, and modified these arrays to create accessory-present arrays and templatepresent arrays by randomly replacing one of the 16 stimuli with the non-cued or the cued colors, respectively. Heterogeneous arrays were created by randomly placing the eight distractor colors on the left eight and right eight positions. Homogeneous search arrays were created by choosing just one of the eight available distractor colors and filling all search stimuli with that color. In the neither-present condition and the accessory-present condition, these arrays required a no response, which was signaled by the participant using the $m$ key. Template present arrays required a yes response, which was signaled by the participant using the $\mathrm{z}$ key. Participants were given a maximum of $4 \mathrm{~s}$ to produce a response. If participants entered an incorrect response, or no response, feedback (i.e., a warning message) was displayed for $2 \mathrm{~s}$.
After a response was given, the memory test display was shown immediately. In this display, white hollow squares appeared in the positions of the memory stimuli from earlier in the trial. One of these squares was drawn with a 1-pixel width, and the other was drawn with a 5-pixel width: the latter was the square for which participants were asked to recall the color. To report the remembered color, participants used the $\mathrm{z}$ and $\mathrm{m}$ keys to move a pointer, $1^{\circ}$ in length, clockwise or counterclockwise, respectively, around the outside of the color wheel $\left(12^{\circ}\right.$ in radius and $0.4^{\circ}$ thick), until the pointer was above the color they thought best matched the color they remembered. Once participants were satisfied with their response, they pressed the space bar to end the trial. Memory responses were again required within $5 \mathrm{~s}$ to ensure the experiment could be completed within the session. If no response was given, participants saw a warning message for $2 \mathrm{~s}$. Participants completed 300 of such trials, with a break every 50 trials. The entire experiment took between 45 and $60 \mathrm{~min}$ to complete.

\section{Results}

As shown in Fig. 2, search performance was worse in the heterogeneous condition than in the homogeneous condition, as expected. Responses on correct trials were slower, $F(1,22)$ $=17.07, p<.001$, and approximately $17 \%$ more errors were made, $F(1,22)=110.2, p<.001$, when distractors were heterogeneous. Search patterns differed for homogenous and heterogeneous searches, $F(2,44)=12.20, p<.001$, with search of heterogeneous arrays being quicker when the template was present, $M=1,055 \mathrm{~ms}, S E=150 \mathrm{~ms}$, than when neither memory color was present, $M=1,093 \mathrm{~ms}, S E=179 \mathrm{~ms}$, or when the accessory was present, $M=1,102 \mathrm{~ms}, S E=172 \mathrm{~ms}$. In contrast, homogeneous searches were fastest on neitherpresent trials, $M=741 \mathrm{~ms}, S E=130 \mathrm{~ms}$, compared to accessory-present trials, $M=895 \mathrm{~ms}, S E=171 \mathrm{~ms}$, and template-present trials, $M=852 \mathrm{~ms}, S E=141 \mathrm{~ms}$, which suggests that deciding whether the unique color matched the template or not incurred a search time cost. Accuracy was also higher on neither-present trials than on both accessory-present trials and target-present trials, $F(2,44)=12.57, p<.001$, meaning that participants sometimes false alarmed to the accessory's presence (about $5 \%$ of trials).

The critical question was whether or not templates would be remembered better than accessory memories in the homogeneous search condition, where guidance to the target was trivially easy, and so template sharpening was not necessary. Our initial analyses quantified memory errors as the reciprocal of the standard deviation $(1 / \sigma)$ of individual color responses from the correct color on each trial following correct search responses without using a modeling approach. We focused our analyses on only the neither-present trials (plotted in Fig. 2), since no priming of either memory representation by stimuli presented in the search display could have occurred on these trials (the same 

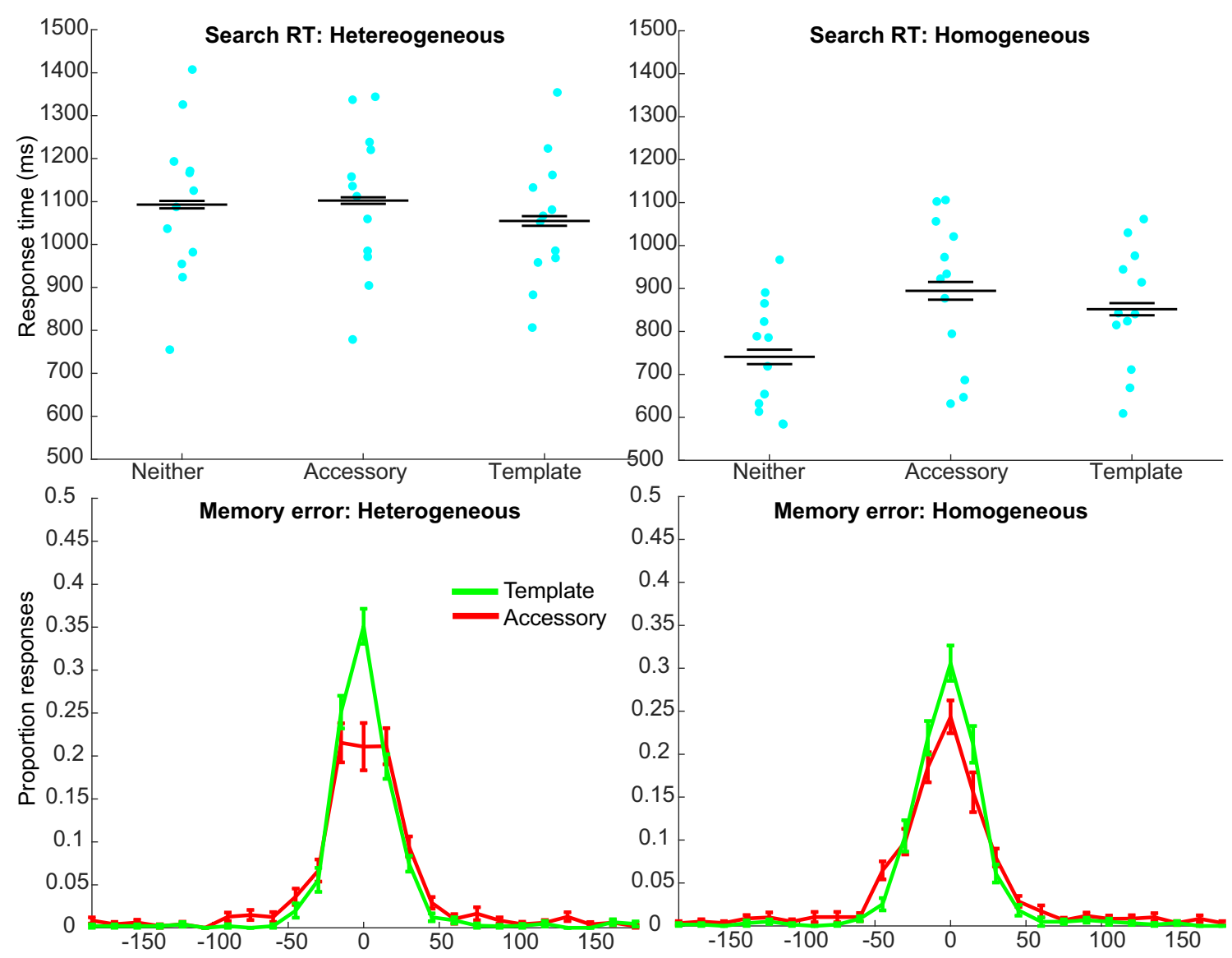

Fig. 2 Upper panels: Search time with heterogeneous distractors (left) and homogeneous distractors (right) as a function of which remembered color was in the search array. Lower panels: Memory error histograms for the heterogeneous distractor (left) and homogeneous distractor (right)

for accessories and templates for searches where neither remembered color appeared during the search. Error bars depict one standard error of the mean

conclusions were reached from a full factorial analysis). Templates were remembered better than accessories, $F(1,22)$ $=22.65, p<.001$, but this did not interact with search type, $F(1$, $22)=0.61, p=.45$. Preplanned comparisons showed that memory for templates was better than memory for accessory items following both a heterogeneous search, $t(11)=3.36, p=.006$, and a homogeneous search, $t(11)=3.37, p=.006$. The fact that a memory difference occurred even when relevant items popped out suggests that improving distractor rejection is not the driving force behind the template memory advantage, and supported the recognition-weighting hypothesis.

We also compared memory performance after modeling individual participants' memory error distributions as a mixture of guesses and target responses (Bays, Catalao, \& Husain, 2009). The estimated standard deviation of recalled colors was smaller (i.e., more precise) for templates than accessory memories, $F(1,22)=8.77, p=.007$, and did not interact with search type, $F(1,22)=1.04, p=.32$. Similarly, the estimated probability that the tested color was in memory (i.e., the height of the tails of the response distribution) was higher for templates than for accessory items, $F(1,22)=8.46, p=.008$, with no modulation by search type, $F(1,22)=0.29, p=.60$. Separating memory error into different error types did nothing to change the conclusions drawn from un-modeled data.

\section{Discussion}

The results of Experiment 1 strongly argue against the adaptive-weighting hypothesis, which holds that participants strategically (or otherwise) prioritize the fidelity of template representations to more efficiently separate targets from distractors. Templates were consistently remembered better than accessory items both when target localization was difficult, because distractors were heterogeneous (Duncan \& Humphreys, 1989), and when target localization was trivially easy, because distractors were homogenous. As such, it seems that the difference in memory fidelity that results when one memory is assigned template status serves some other function than augmenting search guidance.

One limitation of Experiment 1 is that the critical contrast of heterogeneous and homogeneous search was run 
between subjects. In Experiment 2 we sought to make a more direct, within-subjects comparison of the difference between template and accessory memory fidelity following a difficult and an easy search.

\section{Experiment 2}

The goal of Experiment 2 was to compare template and accessory memory fidelity within subjects following different types of search tasks. To collect sufficient data for both search conditions and test memory for the different types of objects, we modified the search task from Experiment 1. Whereas the search task in Experiment 1 required participants to report the presence or absence of the cued object, Experiment 2 used a compound search task (Olivers \& Meeter, 2006) wherein each stimulus in a search array contained a left- or right-tilted line. Participants were told that they needed to find the single colored square that matched the cued item they had stored in memory, and report the orientation of the line inside that square. Every trial contained a single item the color of which exactly matched the template color (i.e., the target), and a single item the color of which exactly matched the accessory color (i.e., a memory-matching non-target). The rest of the search items were either homogeneously colored, during easy search blocks, or heterogeneously colored, during difficult search blocks. In addition to providing a more sensitive within-subjects' measurement of the template advantage, Experiment 2 ensured that all participants experienced both the easy and hard search condition. If experience with a more difficult search is necessary to realize that template sharpening is unnecessary during easy search, then we might see the template advantage disappear here following easy searches.

\section{Method}

\section{Participants}

Twenty-eight participants, none of whom participated in Experiment 1, volunteered for Experiment 2. All provided informed consent before participating and were awarded partial course credit as compensation. Data were collected until 24 participants remained after exclusion criteria were applied.

Four participants were excluded for performance that was not statistically distinguishable from chance in one or more conditions for either the search or memory task. One participant performed the search task at chance levels, two participants had chance-level memory in either the homogeneous or heterogeneous search blocks, and one participant produced chance-level responses in all conditions for both search and memory.

\section{Stimulus and procedure}

Stimuli and procedure were the same as in Experiment 1, with the following exceptions. Search arrays were constructed the same way as in Experiment 1 with two exceptions. First, both the cued and uncued color on each trial replaced a randomly positioned distractor on all trials. Second, all search stimuli were overlaid with black lines tilted $45^{\circ}$ leftwards or rightwards. Participants pressed the $\mathrm{z}$ key to indicate that the target square had a left-tilted line, and pressed the $m$ key to indicate that the target square had a right-tilted line. Search and memory display timeouts were extended to $6 \mathrm{~s}$ so as not to truncate reaction time distributions. All participants completed six pseudo-randomly presented blocks of 50 trials, half of which required search for targets embedded in arrays of homogeneous distractors (with the exception of the non-cued stimulus), and half of which required search for targets embedded in arrays of heterogeneous distractors. Block order was randomized by appending pairs of heterogeneous and homogeneous blocks whose order was randomized, ensuring that no more than two sequential blocks of the same type could be presented. Participants completed 300 blocks in total, allowing for 75 trials in each of the four cells in the design. The experiment took approximately $1 \mathrm{~h}$ to complete.

\section{Results}

As shown in Fig. 3, search was over $400 \mathrm{~ms}$ faster in the homogeneous than in the heterogeneous condition, $F(1,23)$ $=70.74, p<.001$, and led to a $10 \%$ difference in error rate in favor of the homogeneous search condition, $F(1,23)=83.12$, $p<.001$. Participants' error $(1 / \sigma)$ in the memory task once again showed a template fidelity benefit, $F(1,23)=56.21, p<$ .001 , with marginal evidence for a larger benefit after heterogeneous search, $F(1,23)=3.84, p=.06$. Analyzing modeled memory $S D$ provided additional statistical support for this interaction, $F(1,23)=22.78, p<.001$, with poorer precision following heterogeneous distractors (though this was largely driven by accessory memory precision differences between the search conditions), $F(1,23)=18.94, p<.001$, and the familiar template advantage, $F(1,23)=51.44, p<.001$. Estimating the probability of memory based on the height of the tails of the response distributions in color space showed a similar pattern. That is, an ANOVA with the factors of memory type (template vs. accessory item) and search condition (heterogeneous vs. homogeneous) was run on the participants' estimates of Pmem. This yielded a memory type $\mathrm{x}$ search condition interaction: $F(1,23)=5.16, p=.033$, a benefit for templates: $F(1,23)=19.10, p<.001$, but no memory cost due to the type of distractors, $F(1,23)=0.79, p=.38$. Thus, there was some indication that the template-accessory difference was larger during heterogeneous search blocks. However, it was still the case that templates were remembered better than 


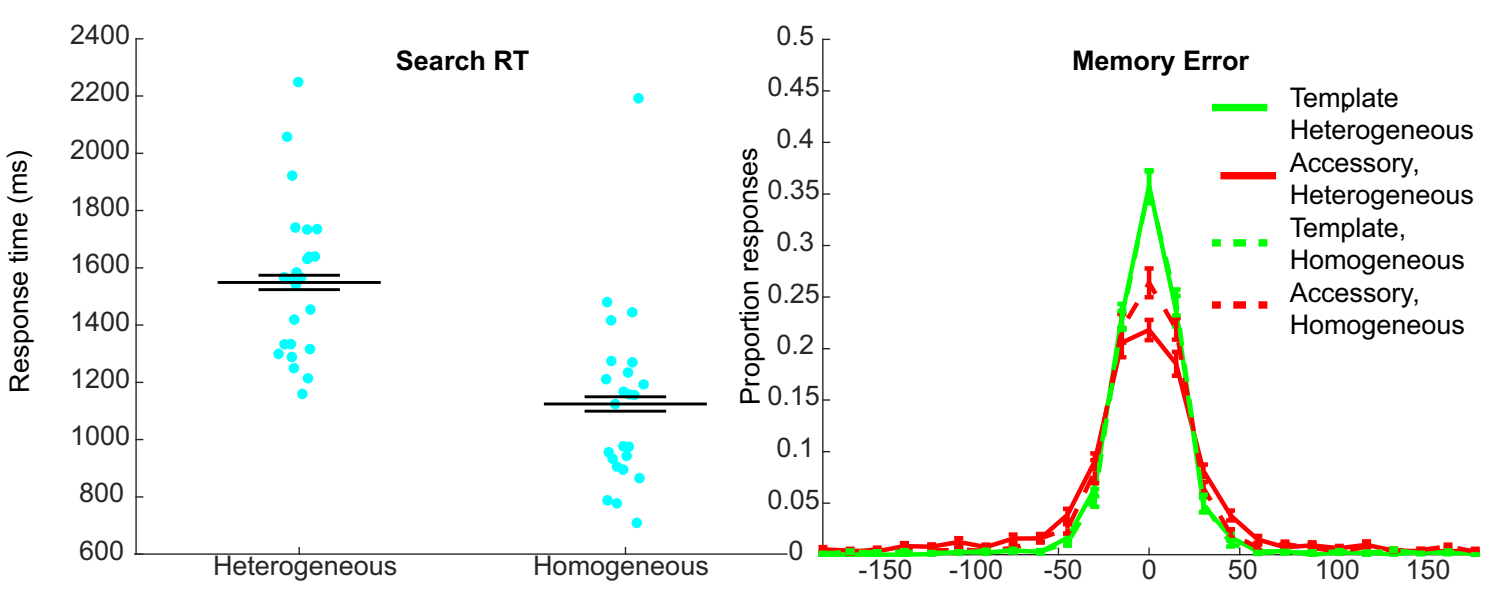

Fig. 3 Left panel: Search time in Experiment 2 when distractors were heterogeneous and homogeneous. Right panel: Memory error histogram for templates and accessories for both distractor types

accessories when distractors were homogeneous, $t(23)=4.25$, $p<.001$, in a planned comparison.

\section{Discussion}

Experiment 2 replicated the evidence from Experiment 1 that target representations are remembered better than other memory representations regardless of the difficulty of the visual search task. Experiment 2 also suggests that heterogeneous distractors impair the precise retention of colors in working memory. Searching through a heterogeneous display of colors seems to have led to a larger difference between the memory for templates and accessories. One possible explanation for this finding is that heterogeneous distractors lead to more memory interference. However, it could also be due to accessories being sharpened more frequently through resampling during homogeneous searches (Woodman \& Luck, 2007). Although both the template and the accessory colors were always presented in search, the accessory color more likely attracted attention in the context of homogeneous distractors, which should increase its feature contrast, compared to heterogeneous distractors, which make the accessory color nonunique. To address this possibility, we conducted Experiment 3 , which used a present-versus-absent search task, more similar to that used in Experiment 1. Comparing template and accessory memory on target-absent trials, where neither color is present in the array, allowed us to measure memory fidelity without the opportunity for resampling.

\section{Experiment 3}

Experiment 3, like Experiment 2, tested participants on both easy (homogeneous distractors) and difficult (heterogeneous distractor) blocks of search. However, to measure the quality of memory for accessories and templates in the absence of perceptual resampling, we returned to a target-present versus target-absent search task, like that used in Experiment 1. To obtain an adequate number of trials, we dropped the accessory-present trials, such that target-absent searches contained neither of the colors being remembered, and target-present searches always contained the template color and not the accessory color. We focused our analyses on the target-absent condition as in Experiment 1, as it should allow us to measure the precision of participants' memories when there is no opportunity to resample the colors being remembered.

\section{Method}

\section{Participants}

Twenty-four undergraduates from Vanderbilt University participated in Experiment 3. All participants provided informed consent before participating, and none of the participants had already taken part in Experiments 1 or 2 .

Of the 24 participants who completed Experiment 3, eight performed the task with chance-level performance in at least one condition. Clearly intermixing the easy and difficult search tasks in the context of a target-present versus -absent search caused participants some difficulty. Chance performance either occurred in the heterogeneous search condition $(n=2)$, in accessory memory recall $(n=3)$, or in both of these two conditions $(n=3)$. This indicates that the excluded participants could not, or did not, successfully manage to simultaneously remember accessory items and successfully pick out the template from search arrays with multiple, often similar, colors.

\section{Stimuli and procedure}

Experiment 3 was identical to Experiment 2, with the exception of the search displays used. The task was a target-present versus -absent search task, and participants were asked to 
report that the cued color was present, using the $\mathrm{z}$ key, or that it was absent, using the $\mathrm{m}$ key. We increased the total number of trials in the Experiment to 384, so that each of the eight possible conditions (distractor type $\times$ memory type $\times$ target presence) contained 48 trials.

\section{Results}

As shown in Fig. 4, target presence had opposite effects on search time for heterogeneous and homogeneous search, $F(1$, $15)=25.96, p<.001$. Target-present responses were faster than target-absent responses for heterogeneous search, $t(15)=$ $4.92, p<.001$, but target-absent responses were faster during homogeneous search, $t(15)=2.20, p=.044$. Accuracy was also higher for homogeneous search, $F(1,15)=177.45, p<$ .001 , by almost $20 \%$, and response times were faster, $F(1,15)$ $=146.67, p<.001$.

As in Experiments 1 and 2, we again found that templates were generally remembered better than accessories, both when distractors were heterogeneous and when they were homogeneous (see Fig. 4). Looking at trials where neither remembered color was shown during search, raw memory accuracy (calculated as $1 / \sigma$ of color error) was better for templates than accessories overall, $F(1,15)=28.85, p<.001$, and memory accuracy was also better overall following search through heterogeneous distractors, $F(1,15)=4.64, p=.05$. However, this was driven by an interaction, $F(1,15)=5.79, p$ $=.029$, such that template memory was better when distractors were heterogeneous than when they were homogeneous. However, recalling that errors were made more often following heterogeneous distractors than homogeneous distractors, it is possible that excluding trials with search errors also excludes trials where the template color happened to be encoded poorly before the cue even appeared, given that imprecise templates would be expected to cause search errors.
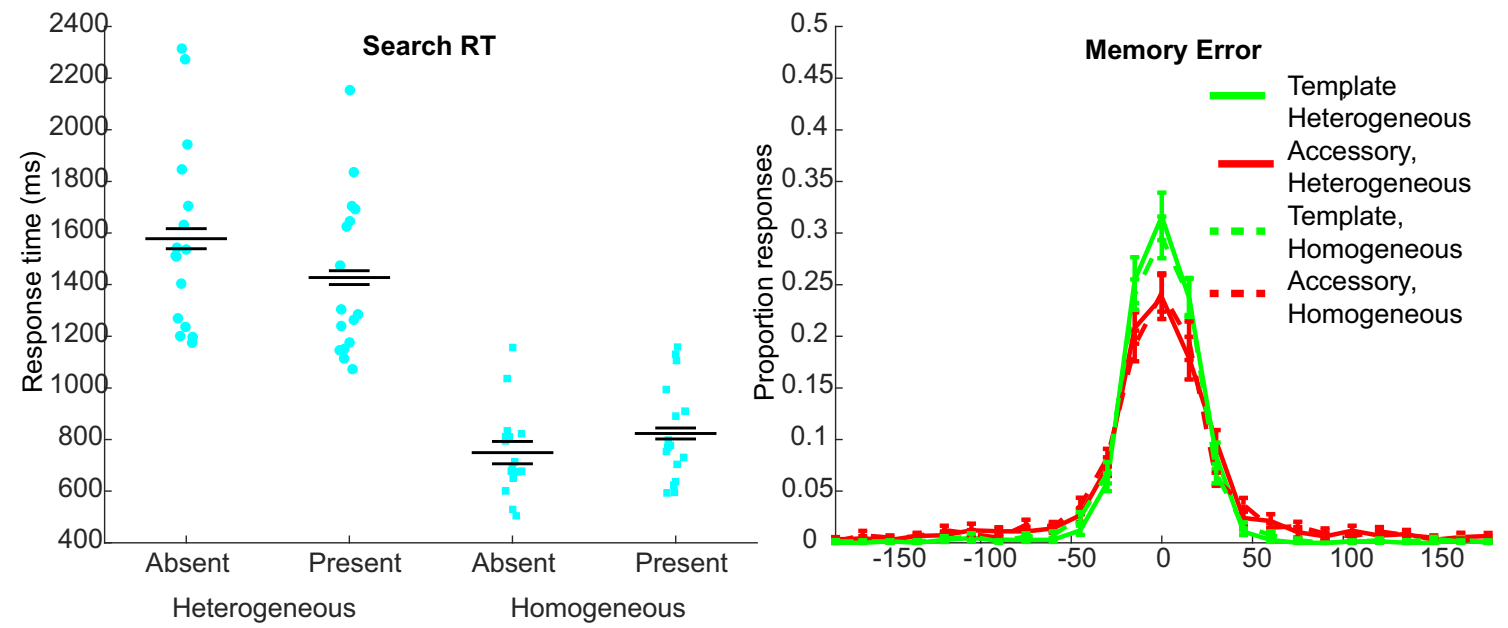

Fig. 4 Left panel: Search time in Experiment 3 as a function of target presence and distractor type (Homogeneous and Heterogeneous). Right panel: Memory error histograms for accessories and templates for both distractor types following target-absent trials 
array of distractors. That is, participants could have learned to respond present whenever there was a singleton, regardless of its color, and still made the correct decision (Bacon \& Egeth, 1994), obviating the need to assign distinct template and accessory statuses to the colors at all. Given that participants weren't informed of this regularity, it may be that they were simply being strategically conservative by following instructions.

\section{General discussion}

When we look for one of two things we are remembering, our memory for what we looked for is better than our memory for what we did not look for (Rajsic et al., 2017). Here, we asked whether this is because we sharpen template memories so that we can later filter out distractors more effectively during search (the adaptive-weighting hypothesis) or because we need to respond affirmatively to a specific feature, once attended, and not others (the recognition-weighting hypothesis). The results of these experiments argue against the former possibility. When we made finding the target trivially easy by presenting the target alongside completely homogeneous distractors, templates were still reported with higher fidelity than accessory memories. While the difference between template and accessory memories was larger following heterogeneous searches in Experiment 2, Experiment 3 demonstrated that this was likely caused by differences in the opportunities for perceptual resampling. On the basis of these results, it seems most sensible to conclude that the template memory advantage we have observed in this task before (Rajsic et al., 2017) reflects the need to make a decision about the template color during search rather than an effort to improve the guidance of attention toward target-defining features and away from distractors during search. We should note as well that template memories could have, in principle, been sharpened during the difficult search as distractors were being rejected, and not in advance of the search. Given that this predicts the same results as the adaptive-weighting hypothesis, it is also inconsistent with our data.

Preparing to use a mental representation for a particular task is not a trivial process. Numerous experiments have now shown that cuing a particular item in a set of already encoded items can improve memory for the cued item compared to other items (Griffin \& Nobre, 2003; see Souza \& Oberauer, 2016 for a review). Our experiments, along with others (Zokaei et al., 2014) help to show that simply using a mental representation can lead to similar differences when a sensitive task (i.e., continuous feature recall) is used to probe the memories themselves. Indeed, instructions to simply think about an item can shift memory performance in favor of those items proportionally to the number of times an item is thought about (Souza, Rerko, \& Oberauer, 2015).
Cued items - those ready to be used - appear to be maintained in a qualitatively different neural state. Lewis-Peacock and colleagues (Lewis-Peacock, Drysdale, Oberauer, \& Postle, 2012; LaRocque, Lewis-Peacock, Drysdale, Oberauer, \& Postle, 2013) have found that the most recently cued item is uniquely decodable from fMRI and EEG. De Vries, van Driel, and Olivers (2017) have also shown that lateralized EEG elicited by items about to be used for search shows stronger alpha suppression contralateral to items that are to be searched for immediately than to items to be searched for later, with no differences in contralateral voltage that reflects visual working memory storage (Vogel \& Machizawa, 2004). These results have been taken to indicate that memory representations currently being used are in a more active state. However, another noteworthy proposal is that cuing a memory for use does more than change the activation state of the memory: it binds the memory to a particular task set in order to prepare for upcoming memory-driven decisions and responses (Myers et al., 2017). This account suggests that benefits for templates could instead result from their being already coupled the relevant decision circuitry for judging whether inputs match that mental representation, as opposed to differences in states of activation.

It is important to stress that the dual memory-search task that we used here was quite difficult. Across each experiment, more participants performed at chance levels than we expected. We take this to indicate that some participants could not encode the two-color memory set with enough precision to reliably distinguish distractors from targets during heterogeneous search. Indeed, some excluded participants showed chance memory of the accessory item only, despite instructions that emphasized the fact that both items could be tested. This may indicate that they dropped the accessory memory in an attempt to remember the template precisely enough to distinguish targets from distractors, as distractors in the heterogeneous condition could often occur from the same color category as the template.

Our results provide an interesting complement to Geng et al.'s (2017) recent demonstration of an improvement in the precision of distractor filtering during search. In contrast, we found almost no role of distractor differences in determining the precision of the attentional template relative to the accessory memory. As noted in the introduction, a major difference between these experiments is whether the target color varied between trials. In our task, template colors changed on every trial, and so participants' only recourse to improving distractor rejection would have been to tune their template using top-down control. In this context, no such special tuning occurred in anticipation of more heterogeneous distractors. On the other hand, experience with irrelevant information does seem to be necessary for improving the allocation of attention away from distractors within a given search array (Cunningham \& Egeth, 2016; Geng et al., 2017; Vatterott \& 
Vecera, 2012). Taken together, these results suggest that more precise distractor rejection requires repeated exposure to relevant and irrelevant visual features, implying that this improved tuning of attention could involve perceptual learning instead of, or in addition to, better cognitive control.

Throughout her iconic work on FIT, Treisman was very sensitive to the possible contribution of feature-based selection strategies to search efficiency (Treisman \& Gelade, 1980; Treisman \& Sato, 1990). For example, in noting the incompatibility between her search efficiency estimates and the convincing demonstration of subset search by Egeth et al. (1984), she concluded that searchers may choose to use feature-based strategies only when they provide frequent enough opportunities for search benefits, anticipating the classic demonstrations of search modes (Bacon \& Egeth, 1994). Although we agree with Treisman that such selection strategies are possible, the results of the experiments we report here provide no evidence that the difference in memory quality between templates and accessory memory representations is a result of such a strategy. Templates were still remembered better than accessories when targets were color singletons, a condition that does not require a featurebased template to separate the target from distractors. We take these results to mean that memory advantages for templates likely do not result from a need to sharpen template memories to improve selection within the search array. Instead, we believe the template memory difference measured in this task reflects the operation of a mechanism that enables decision making specifically, deciding that an attended object is the object being searched for - rather than the signature of a representation that works to shift attention toward target-like objects and away from distractor-like objects.

Acknowledgements This work was supported by grants from the National Institutes of Health (R01-MH110378, R01-EY019882, R01EY025275, and P30-EY08126) and Vanderbilt Chancellor's Fellow Funds.

\section{Compliance with ethical standards}

Competing interests statement The authors declare no competing financial interests.

Open practices statement None of the data or materials for the experiments reported here is available, and none of the experiments were preregistered.

\section{References}

Bacon, W. F., \& Egeth, H. E. (1994). Overriding stimulus-driven attentional capture. Perception \& Psychophysics, 55(5), 485-496. https:// doi.org/10.3758/BF03205306

Bays, P. M., Catalao, R. F. G., \& Husain, M. (2009). The precision of visual working memory is set by allocation of a shared resource. Journal of Vision, 9, 1-11. https://doi.org/10.1167/9.10.7. Introduction
Carlisle, N. B., \& Woodman, G. F. (2011). Automatic and strategic effects in the guidance of attention by working memory representations. Acta Psychologica, 137(2), 217-225. https://doi.org/10.1016/j. actpsy.2010.06.012

Cunningham, C. A., \& Egeth, H. E. (2016). Taming the white bear: Initial costs and eventual benefits of distractor inhibition. Psychological Science, 27(4), 476-485. https://doi.org/10.1177/ 0956797615626564

de Vries, I. E. J., van Driel, J., \& Olivers, C. N. L. (2017). Posterior $\sqcup$ EEG dynamics dissociate current from future goals in working memory-guided visual search. 37(6), 1591-1603. https://doi.org/ 10.1523/JNEUROSCI.2945-16.2016

Desimone, R., \& Duncan, J. (1995). Neural mechanisms of selective visual attention. Annual Review of Neuroscience, 18, 193-222. https://doi.org/10.1146/annurev-psych-122414-033400

Downing, P. E., \& Dodds, C. M. (2004). Competition in visual working memory for control of search. Visual Cognition, 11(6), 689-703. https://doi.org/10.1080/13506280344000446

Duncan, J., \& Humphreys, G. W. (1989). Visual search and stimulus similarity. Psychological Review, 96(3), 433-458. https://doi.org/ 10.1037/0033-295X.96.3.433

Egeth, H. E., Virzi, R. A., \& Garbart, H. (1984). Searching for conjunctively defined targets. Journal of Experimental Psychology. Human Perception and Performance, 10(1), 32-39. https://doi.org/10.1136/ bjsm.2011.084558

Friedman-Hill, S., \& Wolfe, J. M. (1995). Second-order parallel processing: Visual search for the odd item in a subset. Journal of Experimental Psychology. Human Perception and Performance, 21(3), 531-551.

Geng, J. J., Diquattro, N. E., \& Helm, J. (2017). Distractor probability changes the shape of the attentional template. Journal of Experimental Psychology. Human Perception and Performance, 43(12), 1993-2007.

Griffin, I. C., \& Nobre, A. C. (2003). Orienting attention to locations in internal representations. Journal of Cognitive Neuroscience, 15(8), 1176-1194. https://doi.org/10.3758/s13414-011-0218-3

Hout, M. C., \& Goldinger, S. D. (2015). Target templates: The precision of mental representations affects attentional guidance and decisionmaking in visual search. Attention, Perception, \& Psychophysics, 77(1), 128-149. https://doi.org/10.3758/s13414-014-0764-6

Kiyonaga, A., Egner, T., \& Soto, D. (2012). Cognitive control over working memory biases of selection. Psychonomic Bulletin and Review, 19(4), 639-646. https://doi.org/10.3758/s13423-012-0253-7

Kleiner, M., Brainard, D., \& Pelli, D. (2007). What's new in Psychtoolbox-3? Perception 36 ECVP Abstract Supplement, 36, 14. https://doi.org/10.1068/v070821

LaRocque, J. J., Lewis-Peacock, J. A., Drysdale, A. T., Oberauer, K., \& Postle, B. R. (2013). Decoding attended information in short-term memory: An EEG study. Journal of Cognitive Neuroscience, 25(1), 127-142. https://doi.org/10.1162/jocn_a_00305

Lewis-Peacock, J. A., Drysdale, A. T., Oberauer, K., \& Postle, B. R. (2012). Neural evidence for a distinction between short-term memory and the focus of attention. Journal of Cognitive Neuroscience, 24(1), 61-79. https://doi.org/10.1162/jocn_a_00140

Myers, N. E., Stokes, M. G., \& Nobre, A. C. (2017). Prioritizing information during working memory: Beyond sustained internal attention. Trends in Cognitive Sciences, 21(6), 449-461. https://doi.org/ 10.1016/j.tics.2017.03.010

Nakayama, K., \& Silverman, G. H. (1986). Serial and parallel processing of visual feature conjunction. Nature, 320(6059), 264-265.

Olivers, C. N. L., \& Meeter, M. (2006). On the dissociation between compound and present/absent tasks in visual search: Intertrial priming is ambiguity driven. Visual Cognition, 13(1), 1-28. https://doi. org/10.1080/13506280500308101

Olivers, C. N. L., Meijer, F., \& Theeuwes, J. (2006). Feature-based memory-driven attentional capture: Visual working memory content 
affects visual attention. Journal of Experimental Psychology. Human Perception and Performance, 32(5), 1243-65. https://doi. org/10.1037/0096-1523.32.5.1243

Olivers, C. N. L., Peters, J., Houtkamp, R., \& Roelfsema, P. R. (2011). Different states in visual working memory: When it guides attention and when it does not. Trends in Cognitive Sciences, 15(7), 327-34. https://doi.org/10.1016/j.tics.2011.05.004

Pashler, H. (1987). Detecting conjunctions of color and form : Perception \& Psychophysics, 41(3), 191-201.

Peters, J. C., Goebel, R., \& Roelfsema, P. R. (2008). Remembered but unused: The accessory items in working memory that do not guide attention_Peters et al., 2009.pdf. Journal of Cognitive Neuroscience, 21(6), 1081-1091.

Rajsic, J., Ouslis, N. E., Wilson, D. E., \& Pratt, J. (2017). Looking sharp: Becoming a search template boosts precision and stability in visual working memory. Attention, Perception, \& Psychophysics, 79(6). https://doi.org/10.3758/s13414-017-1342-5

Reinhart, R. M. G., \& Woodman, G. F. (2014). High stakes trigger the use of multiple memories to enhance the control of attention, (August), 2022-2035. https://doi.org/10.1093/cercor/bht057

Souza, A. S., \& Oberauer, K. (2016). In search of the focs attention in working memory: 13 years of the retro-cue effect. Attention, Perception, \& Psychophysics, 78, 1839-1860. https://doi.org/10. 3758/s13414-016-1108-5

Souza, A. S., Rerko, L., \& Oberauer, K. (2015). Refreshing memory traces: Thinking of an item improves retrieval from visual working memory. Annals of the New York Academy of Sciences, 1339(1), 2031. https://doi.org/10.1111/nyas.12603

Summerfield, C., \& Koechlin, E. (2008). A neural representation of prior information during perceptual inference. Neuron, 59(2), 336-347. https://doi.org/10.1016/j.neuron.2008.05.021

Treisman, A, \& Sato, S. (1990). Conjunction search revisited. Journal of Experimental Psychology. Human Perception and Performance, 16(3), 459-478. https://doi.org/10.1037/0096-1523.16.3.459

Treisman, A. M., \& Gelade, G. (1980). A feature-integration theory of attention. Cognitive Psychology, 12(1), 97-136. https://doi.org/10. 1016/0010-0285(80)90005-5

Moorselaar, D. Van, Theeuwes, J., \& Olivers, C. N. L. (2016). Learning changes the attentional status of prospective memories. Psychonomic Bulletin \& Review, 1483-1490. https://doi.org/10. 3758/s13423-016-1008-7
Vatterott, D. B., \& Vecera, S. P. (2012). Experience-dependent attentional tuning of distractor rejection. Psychonomic Bulletin and Review, 19(5), 871-878. https://doi.org/10.3758/s13423-012-0280-4

Vogel, E. K., \& Machizawa, M. G. (2004). Neural activity predicts individual differences in visual working memory capacity, 428(April), 1997-2000.

Wolfe, J. M., Cave, K. R., \& Franzel, S. L. (1989). Guided search : An alternative to the feature integration model for visual search, 15(3), 419-433.

Woodman, G. F., Carlisle, N. B., \& Reinhart, R. M. G. (2013). Where do we store the memory representations that guide attention ?, 13, 117. https://doi.org/10.1167/13.3.1.doi

Woodman, G. F., \& Luck, S. J. (2007). Do the contents of visual working memory automatically influence attentional selection during visual search? Journal of Experimental Psychology. Human Perception and Performance, 33(2), 363-77. https://doi.org/10.1037/00961523.33.2.363

Woodman, G. F., Vogel, E. K., \& Luck, S. J. (2001). Visual search remains efficient when visual working memory is full. Psychological Science, 12(3), 219-224. https://doi.org/10.1111/1467-9280.00339

Zelinsky, G. J. (2008). A theory of eye movements during target acquisition. Psychological Review, 115(4), 787-835. https://doi.org/10. 1037/a0013118.A

Zelinsky, G. J., \& Bisley, J. W. (2015). The what, where, and why of priority maps and their interactions with visual working memory, 1339, 154-164. https://doi.org/10.1111/nyas.12606

Zohary, E., \& Hochstein, S. (1989). How serial is serial processing in vision ?, 18(May 1988), 191-200.

Zokaei, N., Ning, S., Manohar, S., Feredoes, E., \& Husain, M. (2014). Flexibility of representational states in working memory. Frontiers in Human Neuroscience, 8(November), 1-12. https://doi.org/10. 3389/fnhum.2014.00853

Publisher's note Springer Nature remains neutral with regard to jurisdictional claims in published maps and institutional affiliations. 